\title{
One-pot ball-milling preparation of graphene/carbon black aqueous inks for highly conductive and flexible printed electronics
}

\author{
Xiao Yang ${ }^{1,2}$, Xiao-Ming $\mathrm{Li}^{1}$, Qing-Qiang Kong ${ }^{1,2}$, Zhuo Liu ${ }^{1}$, Jing-Peng Chen ${ }^{1,2}$, Hui Jia ${ }^{1,2}$, \\ Yan-Zhen Liu ${ }^{1}$, Li-Jing Xie ${ }^{1}$ and Cheng-Meng Chen ${ }^{1 *}$
}

\begin{abstract}
Stable aqueous carbon inks, with graphene sheets (GSs) and carbon black (CB) as conductive fillers, are prepared by a simple one-pot ball-milling method. The asprepared composite ink with $10 \mathrm{wt} \%$ GSs shows optimized rheological properties (viscosity and thixotropy) for screen printing. The as-printed coatings based on the above ink are uniform and dense on a polyimide substrate, and exhibit a sandwich-type conductive three dimensional network at the microscale. The resistivity of the typical composite coating is as low as $0.23 \pm 0.01 \Omega \mathrm{cm}\left(92 \pm 4 \Omega \mathrm{sq}^{-1}, 25 \mu \mathrm{m}\right)$, which is $30 \%$ as that of a pure $\mathrm{CB}$ coating $(0.77 \pm 0.01 \Omega \mathrm{cm})$. It is noteworthy that the resistivity decreases to $0.18 \pm 0.01 \Omega \mathrm{cm}\left(72 \pm 4 \Omega \mathrm{sq}^{-1}\right.$, $25 \mu \mathrm{m})$ after a further rolling compression. The coating exhibits good mechanical flexibility, and the resistance slightly increases by $12 \%$ after 3000 bending cycles. With the CB/GSs composite coatings as a flexible conductor, fascinating luminescent bookmarks and membrane switches were fabricated, demonstrating the tremendous potential of these coatings in the commercial production of flexible electronics and devices.
\end{abstract}

Keywords: graphene, carbon black, conductive inks, printed electronics, one-pot ball-milling

\section{INTRODUCTION}

With the rapid development of the Internet of Things [1], there is increasing demand for lightweight, miniaturized and smart consumer electronics, such as flexible displays [2], sensors [3,4], micro-supercapacitors [5,6], field-effect transistors $[7,8]$ and radio-frequency identification tags $[9,10]$. However, traditional solid-state technology [11] is unsatisfactory for flexible devices because it requires a multistep process and high-cost equipment. Thus, printed electronic technologies [12], including screen, inkjet, gravure and flexographic printing, offer an alternative due to their low cost, simplicity, and high-throughput production. Among these processing technologies, screen printing has unique features such as high compatibility with various conductive inks and substrates as well as high reproducibility [11]. As the core of screen-printed electronic technology [13], functional liquid-phase electronic inks have received extensive attention from the industrial and academic communities. More importantly, the screen inks require a moderate solvent evaporation rate, so the use of less volatile, environmentally friendly water as a solvent is possible [14]. At present, low-cost and high-performance conductive ink is urgently needed [15].

Metallic nanoparticle-based inks are common conductive inks and are widely studied due to their high conductivity of approximately $10^{7} \mathrm{~S} \mathrm{~m}^{-1}$ [16]; however, they also suffer from many issues, including high cost and instability problems such as metal oxidation [17] or silver electromigration [18]. Consequently, the replacement of metal materials with stable, inexpensive, chemically inert and nontoxic carbon-based materials for mass production is of great significance. Carbon black $(\mathrm{CB})$ is a conductive additive that plays a unique role in the formulations of electrode pastes [19], conductive inks [20] and catalyst supports [21]. CB is initially composed of spheroidal or ellipsoidal primary particles, which form aggregates quickly in a CB suspension. The aggregates can bind together through van der Waals forces to form loose agglomerates $(10-100 \mu \mathrm{m})$ [22]. The contact between the aggregates is "point-to-point" contact, resulting in a high contact resistance. Inks made from a single CB filler have high resistivity that limits their application. To increase

${ }^{1}$ CAS Key Laboratory of Carbon Materials, Institute of Coal Chemistry, Chinese Academy of Sciences, Taiyuan 030001, China

${ }^{2}$ University of Chinese Academy of Sciences, Beijing 100049, China

* Corresponding author (email: ccm@sxicc.ac.cn) 
the conductivity of carbon inks, researchers have introduced other one-dimensional (1D) conductive fillers, such as carbon nanotubes (CNTs) and carbon nanofibers (CNFs). Unfortunately, CNTs are prone to entangling with adjacent CNTs, making it very difficult to disperse CNTs well in the polymer matrix of inks [23]. For CNF bundles, the electrons flow through the outermost fibers of the bundles, while the inner fibers contribute less to their conductivity [24]. Compared with the characteristics of CNFs and CNTs, graphene sheets (GSs), novel 2D flexible honeycomb networks, composed of monolayer $\mathrm{sp}^{2}$-hybridized carbon atoms, possess high carrier mobility $\left(15,000 \mathrm{~cm}^{2} \mathrm{~V}^{-1} \mathrm{~s}^{-1}\right.$ under ambient conditions) [25], conductivity $\left(2000 \mathrm{~S} \mathrm{~cm}^{-1}\right)$ [26], chemical stability [27] and intrinsic flexibility [28]. A large number of studies [29-33] have shown that graphene-based conductive inks have a high conductivity up to $4.3 \times 10^{4} \mathrm{~S} \mathrm{~m}^{-1}$ $\left(0.912 \Omega \mathrm{sq}^{-1}, 25 \mu \mathrm{m}\right)$ [10]. GSs are promising candidates for carbon-based inks, especially for printed flexible electronics [14].

The formulation design of $\mathrm{CB} / \mathrm{GSs}$ composite inks also draws inspiration from the literature precedent in the field of energy storage. Compared with the "point-topoint" contact of CB, the "plane-to-point" contact of GSs and $\mathrm{CB}$ enables high interfacial contact efficiency. In this context, Su et al. [19] used CB and graphene as electronconducting additives in $\mathrm{LiFePO}_{4}$-based cathodes in lithium-ion batteries, and found that the bulk ohmic resistance $\left(R_{\mathrm{b}}\right)$ of the $\mathrm{CB} /$ graphene-modified $\mathrm{LiFePO}_{4}$ cell $(11.7 \mathrm{~m} \Omega)$ was lower than that of a commercial cell $(16.0 \mathrm{~m} \Omega)$, and this difference was attributed to the more effective electron transport network created with $\mathrm{CB}$ and graphene. In addition, $\mathrm{CB}$ and graphene were used to modify aluminum foil (as a cathode current collector) to enhance the electrical contact and charge transport between electrode materials and current collectors in lithium-ion batteries [34]. Furthermore, in the preparation of piezoelectric films, the addition of a moderate amount of $\mathrm{CB} /$ few-layer graphene (FLG) to a poly(vinylidene fluoride)-co-hexafluoropropylene (PVDF-co-HFP) hybrid composite film can enhance the conductivity of the nanofiller network [35]. There are also studies on graphene/ CB composite inks for printed electronics. Ji et al. [36] prepared inkjet ink using reduced graphene oxide (rGO) and $\mathrm{CB}$ as fillers, and the resulting flexible paper-based circuits displayed a resistivity of $66.1 \Omega \mathrm{cm}$ $\left(2.6 \times 10^{4} \Omega \mathrm{sq}^{-1}, \quad 25 \mu \mathrm{m}\right)$. Additionally, graphene/polypyrrole/CB conductive ink was fabricated by the in situ polymerization of pyrrole monomers and GO [37]. However, the resistivity of this ink cannot satisfy the demands of high-performance electronics. Furthermore, understanding the structure-conductivity-printability relationships of screen-printing conductive ink remains a challenge.

Herein, we report the optimal formulation and direct printing of high-performance $\mathrm{CB} / \mathrm{GSs}$ composite aqueous conductive inks. Composite inks with different proportions of GSs and CB were designed for screen printing. Moreover, the rheological properties and stability of the inks, and the microstructure, 3D surface morphologies and mechanical flexibility of the printed patterns were characterized. A luminescent bookmark and membrane switch fabricated from the CB/GSs composite printed coatings suggest new possibilities for their applications in electronics and devices.

\section{EXPERIMENTAL SECTION}

\section{Materials}

CB with a diameter of approximately $20-50 \mathrm{~nm}$ was purchased from Tianjin Lihuajin Chemical Industry Co., Ltd. (China). The GSs originated from the Institute of Coal Chemistry, Chinese Academy of Sciences. Poly(acrylic acid) (PAA, $\left.M_{\mathrm{w}}=70,000\right)$ and sodium polyacrylate (P-19) were obtained from Shandong Baoerya Chemical Technology Co., Ltd and Shanghai Wenhua Chemical Pigment Co., Ltd. Airase 5200 and XR-501 were provided by Air Products Asia and Shanghai Xirun Chemical Technology Co., Ltd. Carboxyl methyl cellulose (CMC, $\left.20 \mathrm{~g} \mathrm{~L}^{-1}\right)$ and 1,2-propylene glycol $\left(\mathrm{C}_{3} \mathrm{H}_{8} \mathrm{O}_{2}, 99.5 \%\right)$ were supplied by Daicel Corporation (Osaka, Japan) and Aladdin Chemistry Co., Ltd.

\section{GSs/CB composite ink preparation}

The preparation of the GSs/CB composite ink was similar to that in our previous report [38]. GSs and CB conductive fillers with mass ratios of 0:100, 5:95, 10:90 and 15:85 were dried for $6 \mathrm{~h}$ in an oven. A homogeneous mixture of PAA (26.28 wt.\%), P-19 (24 wt.\%), Airase 5200 (0.45 wt.\%), XR-501 (0.48 wt.\%) and CMC aqueous solution (11.33 wt.\%) was prepared by the magnetic stirring method. Then, a solution (53.06 wt.\%) of deionized water and 1,2-propylene glycol at a weight ratio of 9:1 was prepared. The above dried fillers and the two as-prepared mixtures were poured into the ball-grinding tank. The mixed materials in the planetary ball mill (Tencan Powder Technology Co., Ltd. Changsha, China) equipped with zirconia grinding jars $(500 \mathrm{~mL})$ and zirconia balls $(227 \mathrm{~g}$, diameters of 10 and $5 \mathrm{~mm}$ ) were milled at a rotation speed of $300 \mathrm{rpm}$ for $2.5 \mathrm{~h}$. After ball-milling, the 
obtained inks were poured through a mesh sieve (70 mesh) to remove the larger agglomerates and the zirconia balls. Then, the inks were decanted into a vacuum mixer to remove the bubbles at a rotation speed of $300 \mathrm{rpm}$ for $10 \mathrm{~min}$ (vacuum of $-0.8 \mathrm{bar}$ ). For simplicity, the ink samples were named G/C-0, G/C-5, G/C-10, and G/C-15 according to the GSs content.

\section{Screen printing}

A polyimide (PI) film $(15 \mathrm{~cm} \times 6 \mathrm{~cm} \times 0.0125 \mathrm{~cm})$ was washed with alcohol and used as a printing substrate. A polyester stencil (100-mesh) was placed above the PI film. Then, the inks were poured on top of the stencil and spread with a polyurethane squeegee (13-cm-long, 70-75 Shore hardness) at a $45^{\circ}$ angle. The inks penetrated the openings of the stencil to form a designed pattern. The patterns were a square $(4 \mathrm{~cm} \times 4 \mathrm{~cm}$; length $\times$ width $)$ and a line $(10 \mathrm{~cm} \times 2 \mathrm{~mm}$; length $\times$ width). Finally, the printed patterns were placed in a vacuum oven at $85^{\circ} \mathrm{C}$ for $1 \mathrm{~h}$.

\section{Characterization}

The rheological behavior of the inks was analyzed by a rotational rheometer (MARS60, Haake, Germany) equipped with a plate-plate rotor. All measurements were performed at $25^{\circ} \mathrm{C}$, and the temperature was controlled by a Peltier system. The gap between the parallel plates (plate diameter of $35 \mathrm{~mm}$ ) was $1 \mathrm{~mm}$. To eliminate the shear history, the inks were pre-sheared at a shear rate of $5 \mathrm{~s}^{-1}$ for $1 \mathrm{~min}$, and the rheological tests were started after the inks were allowed to rest for $5 \mathrm{~min}$. The steady-shear rheology was characterized by the viscosity curve (dependence of the apparent viscosity $(\eta)$ on the shear rate $(j))$ acquired over a shear rate range of $0.01-1000 \mathrm{~s}^{-1}$. Then, dynamic rheological measurements, including strain sweep and frequency sweep tests were performed. The strain sweep test was conducted over a strain $(\gamma)$ range from $0.01 \%$ to $100 \%$ at $1 \mathrm{~Hz}$ to determine the linear viscoelastic region (LVR). The frequency sweep test was conducted within a frequency range of $0.1-100 \mathrm{~Hz}$ at a constant strain of $0.05 \%$, which guaranteed that the inner structure of the inks was at a steady state. To further evaluate the thixotropic behavior of the inks and simulate the rheological behavior of the inks during printing, three-interval thixotropy tests (3ITTs) were conducted. First, a shear rate of $5 \mathrm{~s}^{-1}$ was applied for $25 \mathrm{~s}$. Then, the shear rate was maintained at $1000 \mathrm{~s}^{-1}$ for $10 \mathrm{~s}$. Finally, the shear rate was decreased to $5 \mathrm{~s}^{-1}$ for $175 \mathrm{~s}$. Scanning electron microscopy (SEM, Merlin Compact 8010, Zeiss, Germany) and confocal laser scanning microscopy (CLSM, Optelics C130, Lasertec Corp., Yokohama, Japan) were employed for morphological observation of the printed patterns. The thickness and electrical resistivity of the printed conductive patterns were obtained by a thickness gauge (precision of $0.001 \mathrm{~mm}$ ) and a four-point probe resistivity measurement system (RTS-9, Four Probes Tech, China), respectively. The mechanical flexibility was measured using a homemade bending resistance instrument.

\section{RESULTS AND DISCUSSION}

The composite GSs/CB inks were prepared by a simple ball-milling method, and the patterns were printed through screen printing (Fig. 1a). SEM images of the GSs and $\mathrm{CB}$ are shown in Fig. S1. The sandwich-like network structure was obtained by combining GSs and CB. The presence of GSs enhances the stability of GSs/CB inks. The settlement test reveals that pure $\mathrm{CB}$ ink produced some supernatant upon settling, whereas there are no precipitates in the GSs/CB inks after settling for 7 or even 30 days (Fig. 1b-d), which may be due to the mutual dispersion of GSs and CB in the polymer matrix.

Moreover, the rheology of the inks, which depends on the ink formula and processing conditions, guides the selection of the printing method. Therefore, we designed a series of conductive inks with different GS contents, and studied the effect of the $\mathrm{CB} / \mathrm{GSs}$ ratio on the rheological properties of the inks. The screen-printing process has viscosity requirements for inks at various stages, including storage, printing and ink drying. The inks must maintain high viscosity without leakage before printing and then quickly undergo shear thinning and penetrate the screen stencil after the doctor blade is moved. The viscosity curve (Fig. 2a) reflects the flow characteristics of the inks at different shear rates. All samples show pseudoplastic behavior, where the viscosity decreases with increasing shear rate. The $\mathrm{CB} / \mathrm{GSs}$ inks show increased viscosity with increasing GS content. When the mass ratio of GSs increases slightly, the effective volume fraction increases significantly, as the GSs hinder the flow of CB particles during shearing, causing an increase in viscosity. In addition, the inks produce a three-stage flow curve: when the shear rate is less than $0.1 \mathrm{~s}^{-1}$ (region I), the viscosity of G/C-0 decreases slower than that of the $\mathrm{CB} /$ GSs composite inks, which may be because large CB aggregates can resist small external deformation, while the added graphene is sensitive to external deformation [39]. At intermediate shear rates, the viscosity of G/C-0 declines more rapidly than the viscosity of the $\mathrm{CB} / \mathrm{GSs}$ composite conductive inks (region II). In this stage, the large $\mathrm{CB}$ aggregates in $\mathrm{G} / \mathrm{C}-0$ are prone to break up into 


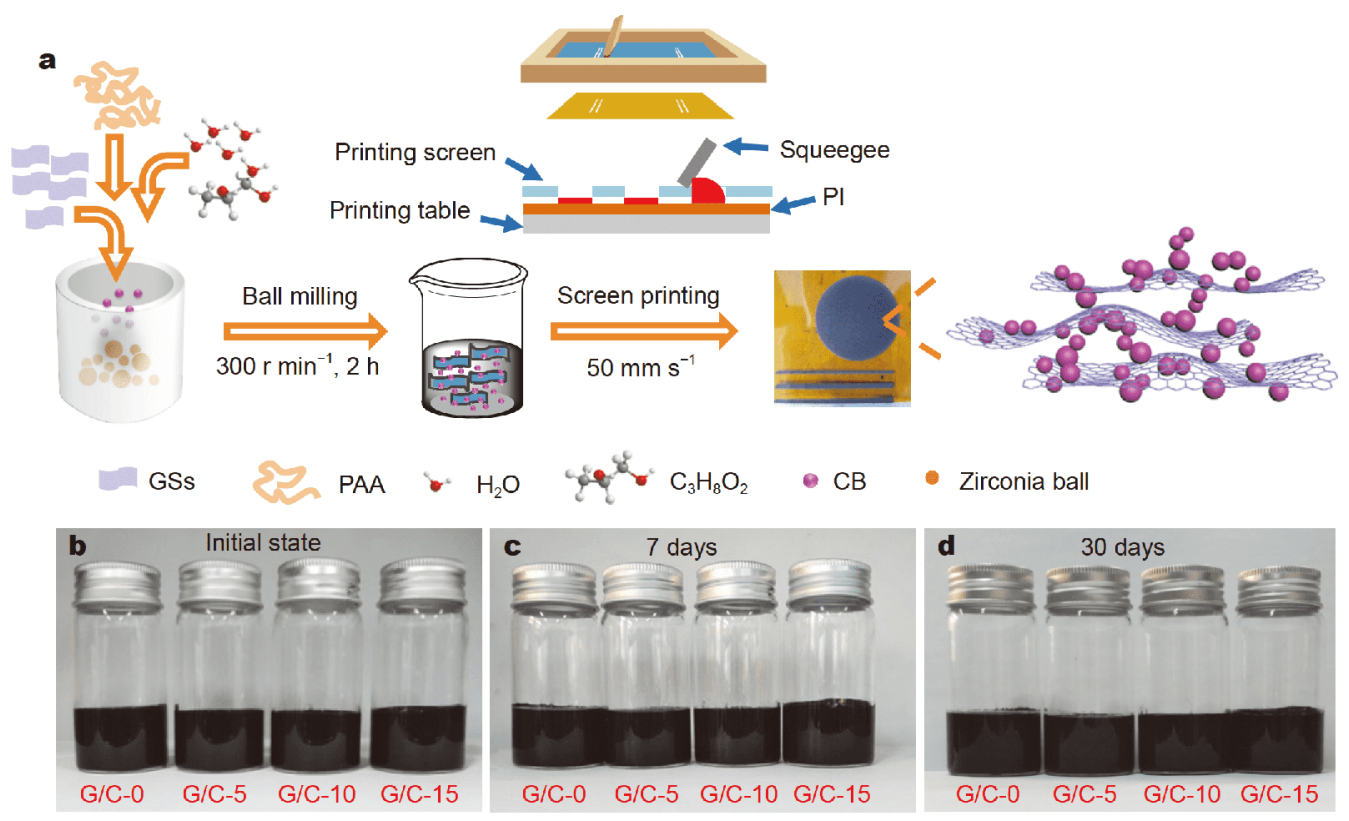

Figure 1 (a) Schematic illustration of the ink preparation and screen-printed pattern preparation processes. (b-d) Optical photographs of the inks in the initial state and after standing for 7 and 30 days, respectively.

smaller aggregates; however, the composite conductive 3D network can effectively resist large deformation. At very high shear rates, the viscosity of all samples quickly descends to a plateau (region III), implying breakdown of the conductive network or the presence of much smaller aggregates that cause the viscosity to decrease [22].

To determine the LVR of the inks, a strain amplitude sweep test was performed, as shown in Fig. S2. Before the critical strain $\left(\gamma_{c}\right)$, the LVR decreases as the graphene mass fraction increases, revealing a decrease in reversible deformation. After exceeding the critical strain, the inks exhibit liquid-like behavior where the viscous modulus $\left(G^{\prime \prime}\right)$ is higher than the storage modulus $\left(G^{\prime}\right)$. The minimal critical strain of the above samples is $0.05 \%$; hence, the dynamic frequency sweep test of the $\mathrm{CB} / \mathrm{GS}$ s composite conductive inks was measured at a strain of $0.05 \%$ (see Fig. 2b). In all frequency ranges, $G^{\prime}>G^{\prime \prime}$ and the damping factor $(\tan \delta)$ are $<1$ (see Fig. S2b), and thus, the inks exhibit solid-like behavior. This highly elastic behavior may be derived from the formation of a percolating gel network [24]. At low frequencies, $G^{\prime}$ and $G^{\prime \prime}$ are largely independent of the frequency. The frequency plateau increases with increasing GS content, indicating that the interaction between $\mathrm{CB}$ and GSs is stronger in the $\mathrm{CB} /$ GSs composite inks with a higher GS content. By contrast, at high frequencies, $G^{\prime}$ and $G^{\prime \prime}$ are dependent on the frequency and both increase with increasing frequency, as the structure of the inks does not have enough time to relax [40]. Moreover, there is a gradual rise in $\tan \delta$ with increasing GS content as a result of the increased viscosity of inks [41]. These results are contributed to the storage and screen printing of composite inks: the inks have higher elasticity in the static state and higher viscosity during printing.

As we can see from the dynamic frequency sweep and steady-shear viscosity curves, the $G^{\prime}$ and viscosity of the ink increase with the increase in the graphene content. To find a suitable formulation for screen printing, we conducted a 3ITT to simulate the viscosity change and observe the deformation and recovery of the ink structure during screen printing. The recovery of the viscosity of the inks after $10 \mathrm{~s}$ of high shear $\left(1000 \mathrm{~s}^{-1}\right)$ occurs increasingly quickly as the GS content increases, indicating that the addition of GSs can enhance the thixotropy of the inks. The statistical time to restore $80 \%$ of the initial viscosity was 47,12 and $5 \mathrm{~s}$ for G/C-0, G/C-5, and G/C-10 (Fig. 2c-e), respectively. In conclusion, the rheological properties of G/C-10 are more suitable for screen printing than those of the other $\mathrm{CB} / \mathrm{GSs}$ composite conductive inks. Although fast viscosity recovery is beneficial for obtaining high-resolution printed patterns, the stress-induced structure regeneration results in a strong colloidal structure. Once the graphene content increases to $15 \mathrm{wt} . \%$ (Fig. 2f), the viscosity of G/C-15 after high shear is too high for the ink to flow flat and form a uniform coating. Similar results have been mentioned in previous reports 

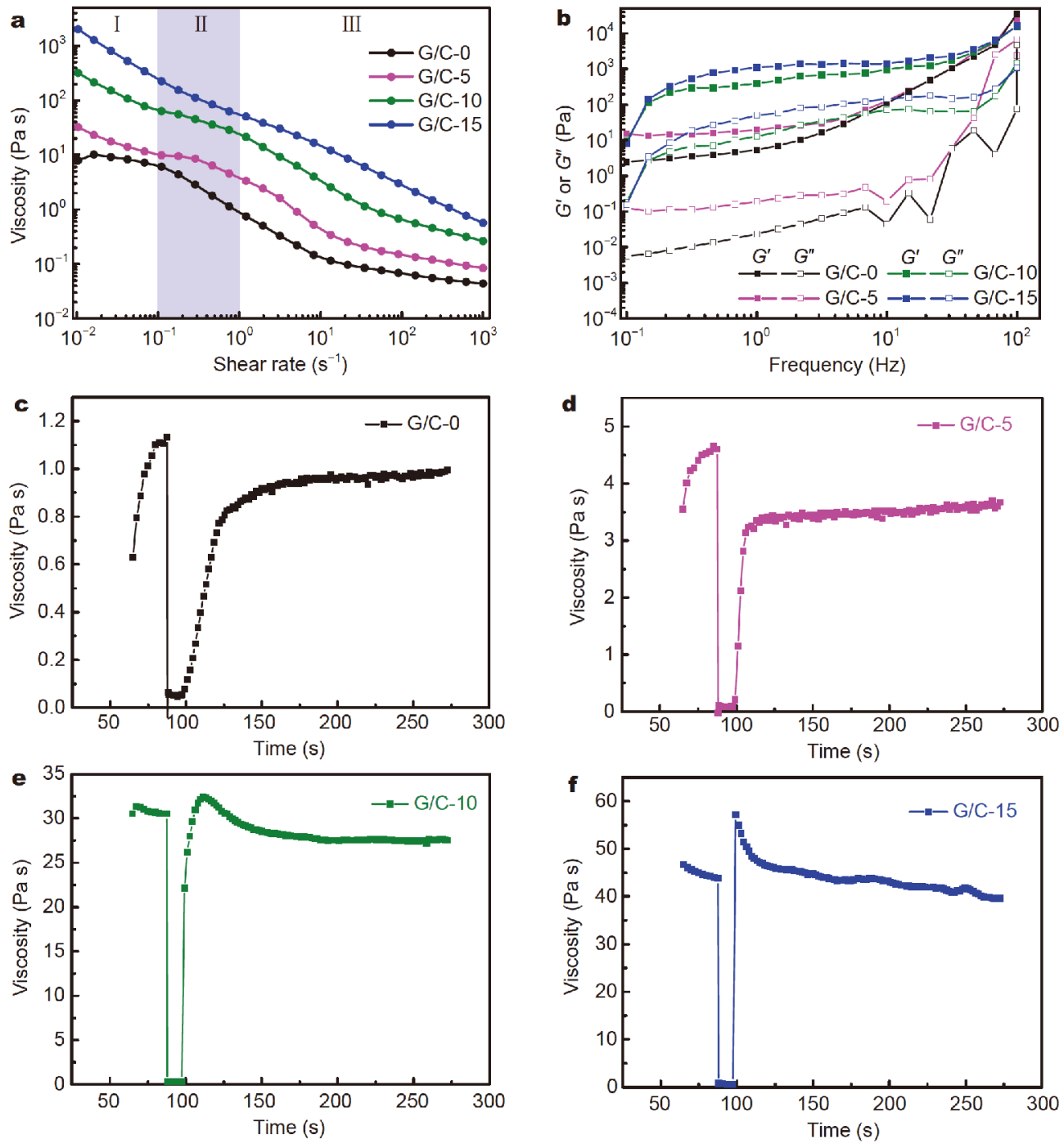

Figure 2 (a) Viscosity plotted as a function of shear rate for different inks. (b) Dynamic frequency sweep for CB/GSs composite conductive inks. (c-f) Viscosity versus time for G/C-0, G/C-5, G/C-10 and G/C-15 in 3ITTs.

[42].

Capillary number $(\mathrm{Ca})[43]$ is an important parameter to describe the wettability of inks and measure printing conditions in printing fields [44]. When the printing speed is $50 \mathrm{~mm} \mathrm{~s}^{-1}$, the shear rate calculated from Equation (1) is $5000 \mathrm{~s}^{-1}$. The corresponding approximate viscosities of inks G/C-0, G/C-5, G/C-10 and G/C-15 are $0.04,0.08,0.26$ and $0.56 \mathrm{~Pa}$ s, respectively. As shown in Table 1, the surface energies of inks G/C-0, G/C-5, G/C10 and G/C-15 are 51.142, 57.718, 58.483 and $63.253 \mathrm{mN} \mathrm{m}^{-1}$, respectively and the Ca values calculated from Equation (2) are $0.039,0.069,0.222$ and 0.443 , respectively. The horizontal flux after screen printing is related to $\mathrm{Ca}$ [45]. The horizontal flux increases with $\mathrm{Ca}$ increasing, which is consistent with the trend in coating thickness.

$\dot{\gamma}=\frac{U}{d}$,

$\mathrm{Ca}=\frac{\eta \times U}{\sigma}$,

where $j$ is the shear rate, $U$ is the printing speed, $d$ is the distance between the tip of the doctor blade and the substrate in the printing process, $\eta$ is the apparent viscosity and $\sigma$ is the surface tension of the ink.

After screen printing, each of the conductive patterns exhibits a compact and continuous coating surface (Fig. 3a-d). We further observed the surface morphology of coatings with higher magnification. In the pure $\mathrm{CB}$ 
Table 1 The surface energy, $\mathrm{Ca}$ and coating thickness of samples G/C$0, \mathrm{G} / \mathrm{C}-5, \mathrm{G} / \mathrm{C}-10$ and $\mathrm{G} / \mathrm{C}-15$

\begin{tabular}{cccc}
\hline Sample & $\begin{array}{c}\text { Surface energy } \\
\left(\mathrm{mN} \mathrm{m}^{-1}\right)\end{array}$ & Ca & $\begin{array}{c}\text { Coating } \\
\text { thickness }(\mu \mathrm{m})\end{array}$ \\
\hline G/C-0 & 51.142 & 0.039 & $9.67 \pm 2.3$ \\
G/C-5 & 57.718 & 0.069 & $9.9 \pm 2.0$ \\
G/C-10 & 58.483 & 0.222 & $9.87 \pm 1.8$ \\
G/C-15 & 63.253 & 0.443 & $10.23 \pm 2.6$ \\
\hline
\end{tabular}

coating, CB particles (20-50 $\mathrm{nm}$ in diameter) are connected to each other and form aggregates (Fig. $3 e$ and i) due to the high surface energy. GSs bridge the CB aggregates when the graphene content is at least $5 \mathrm{wt} . \%$, and we define this structure as a primary conductive network (Fig. 3f). The high carrier mobility of graphene facilitates electron transport between CB agglomerates. It is worth noting that as the graphene content increases to $10 \mathrm{wt} . \%$, the conductive fillers form a well-dispersed primary network (Fig. 3g). As the GSs are further exfoliated, the $\mathrm{CB}$ particles act as spacers to prevent GSs aggregation. The synergistic effect of GSs and CB promotes mutual dispersion of the fillers. We call the microcosmic network
(Fig. 3k) a secondary network (this structure cannot be observed in Fig. 3j). The introduction of 2D GSs increases the contact area of the conductive fillers, and the "pointto-point" contact between CB particles becomes a "planeto-point" contact between GSs and CB, which is consistent with previous reports [46]. Therefore, the number of effective electron transport pathways increases. However, when the GS content increases to $15 \mathrm{wt} . \%$, the content of $\mathrm{CB}$ is not enough to fill the large gaps formed by the overlap of GSs, which causes voids of approximately $1 \mu \mathrm{m}$ and blocks the flow of electrons, as shown in Fig. $3 \mathrm{~h}$, resulting in a high contact resistance.

CLSM was used to characterize the 3D surface morphology of the printed patterns, as displayed in Fig. S3. As the GS content increases, the surface roughness of the coatings increases due to the irregular orientation and inplane bending of the GSs. Combined with the rheological analysis results, these findings show that the addition of GSs facilitates the formation of conductive gel networks. However, the increased thixotropy is not conducive to the formation of smooth coatings. In particular, when the GS content increases to $15 \mathrm{wt} . \%$, square nets are generated from the polyester screen in the coating, as shown in
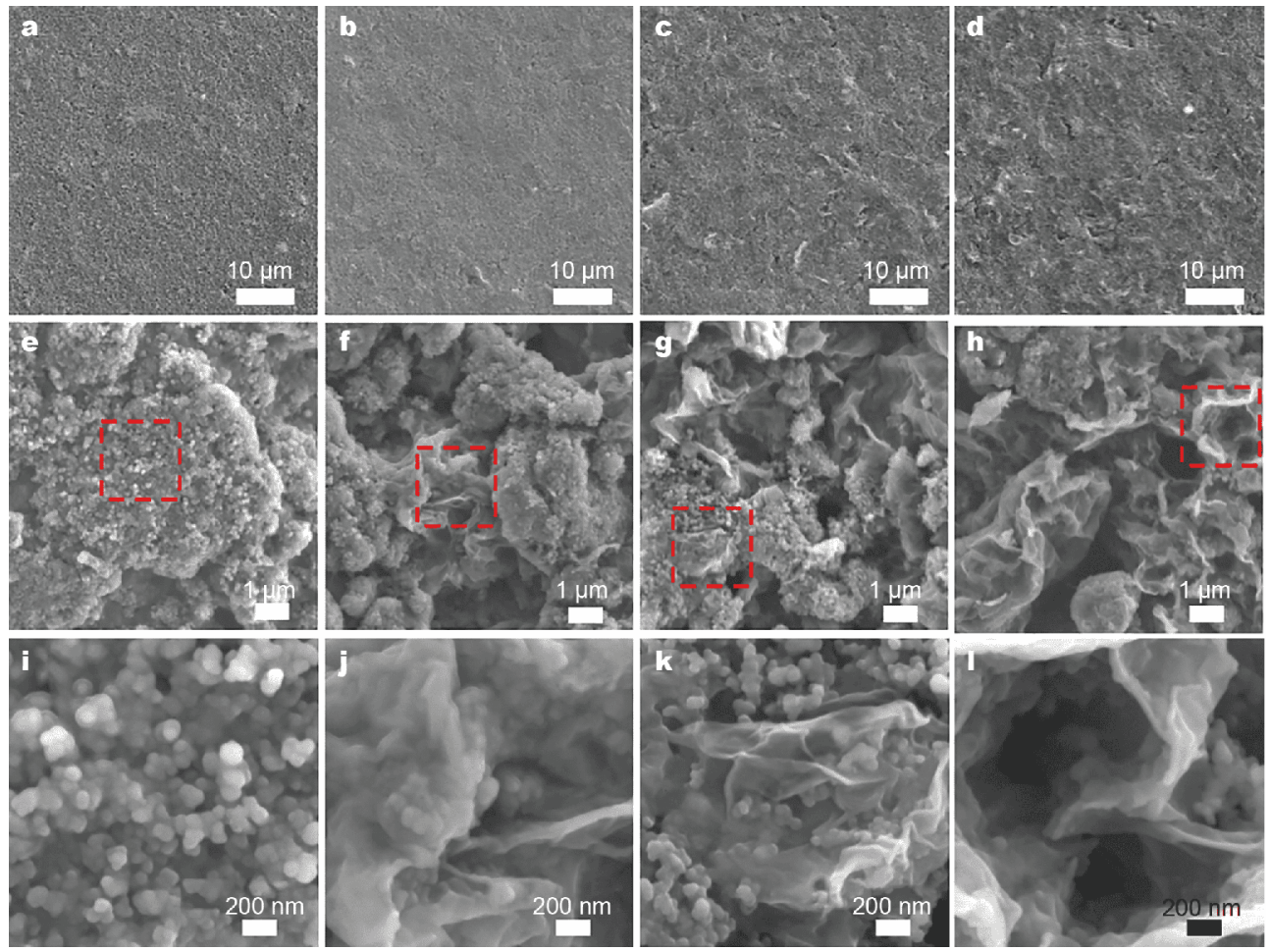

Figure 3 Surface SEM images of (a) G/C-0, (b) G/C-5, (c) G/C-10 and (d) G/C-15 coatings at 5000× magnification. Surface SEM images of (e) G/C-0, (f) G/C-5, (g) G/C-10 and (h) G/C-15 coatings at 20,000× magnification. Surface SEM images of (i) G/C-0, (j) G/C-5, (k) G/C-10 and (l) G/C-15 coatings at $100,000 \times$ magnification. 
Fig. S3d, indicating a poor ability of the inks to flow after screen printing, which is consistent with the 3ITT results.

To explore the influence of the GS content on the resistivity of the coating, we selected 9 points on each coating sample to measure the resistivity using the fourpoint probe method. With increasing GS content, the resistivity first decreases significantly and then increases (Fig. 4). According to Equation (3), as the amount of added GSs increases, the diameters of the contact spot $(d)$ and contact spot area $(\alpha)$ increase. This effect facilitates the mutual dispersion of the CB and GSs, and optimizes the conductive network of the coating. The total resistivity of the coatings decreases. However, when the added GS content continues to increase, the electrical resistivity begins to increase. There are likely two main reasons for this behavior: first, the relative volume fraction of $\mathrm{CB}$ is reduced, becoming insufficient to fill the voids caused by the overlap of GSs; second, the thixotropy of the ink increases, which results in unsmooth channels for effective electron transport. Therefore, the optimal content of GSs is $10 \mathrm{wt} . \%$, and the corresponding resistivity is as low as $0.23 \pm 0.01 \Omega \mathrm{cm}$.

$R=R_{\mathrm{i}}+R_{\mathrm{c}}=R_{\mathrm{i}}+R_{\mathrm{cr}}+R_{\mathrm{t}}=\frac{\rho_{\mathrm{cr}}}{d}+\frac{\rho_{\mathrm{t}}}{a}$,

where $R, R_{\mathrm{i}}$ and $R_{\mathrm{c}}$ are the total resistance of the coating, the intrinsic filler resistance and the contact resistance,

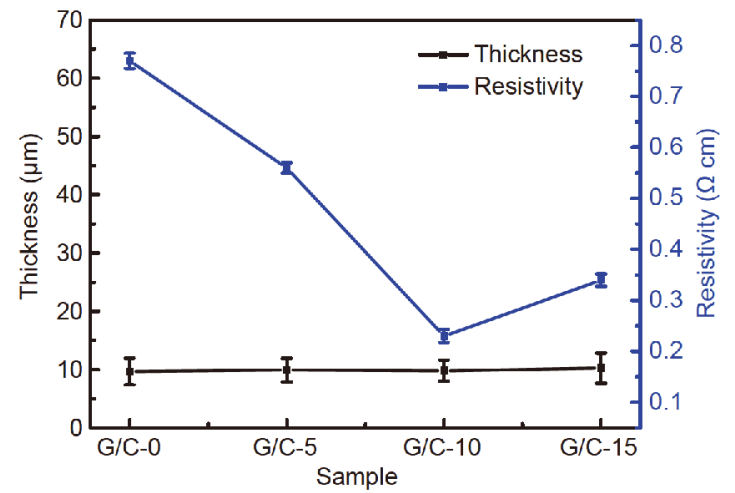

Figure 4 Electrical resistivity variation of printed patterns of G/C-0, G/ C-5, G/C-10 and G/C-15 coatings.

respectively. $R_{\mathrm{cr}}$ and $R_{\mathrm{t}}$ are the constriction resistance and tunneling resistance, respectively. $\rho_{\text {cr }}$ and $d$ are the constriction resistivity and diameter of the contact spot, respectively. $\rho_{\mathrm{t}}$ and $\alpha$ are the tunneling resistivity and contact spot area, respectively [47].

Subsequently, the G/C-10 coating was treated by rolling compression. According to the SEM results, the surface of the as-deposited composite coating exhibits a rough and irregular architecture (Fig. 5a). As shown in the crosssectional SEM images, the GSs and CB are mixed together to form a sandwich-like structure (Fig. 5c), and most of
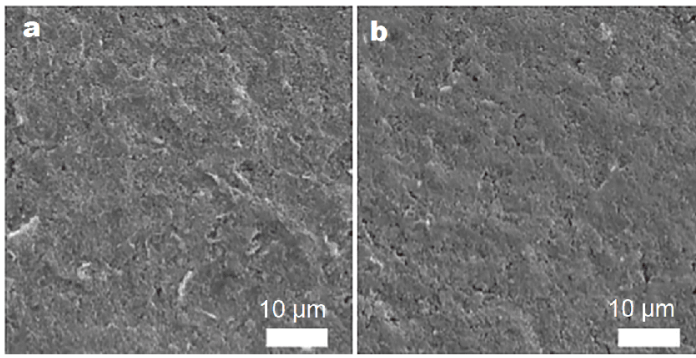

e

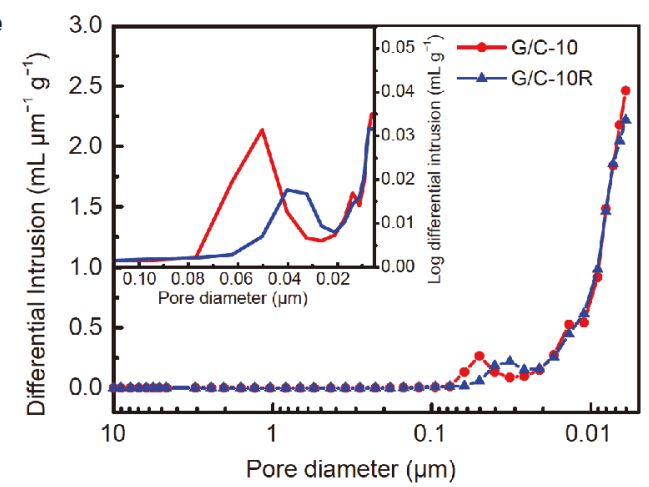

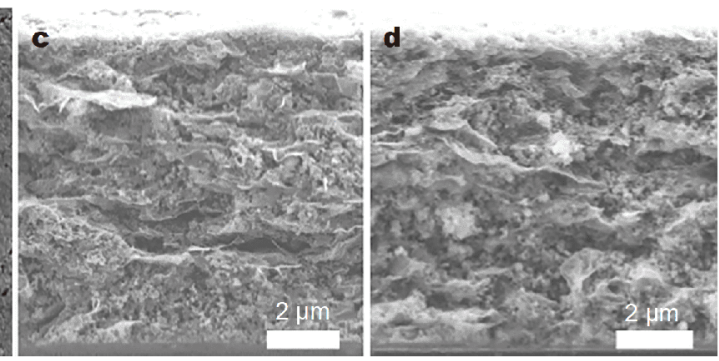

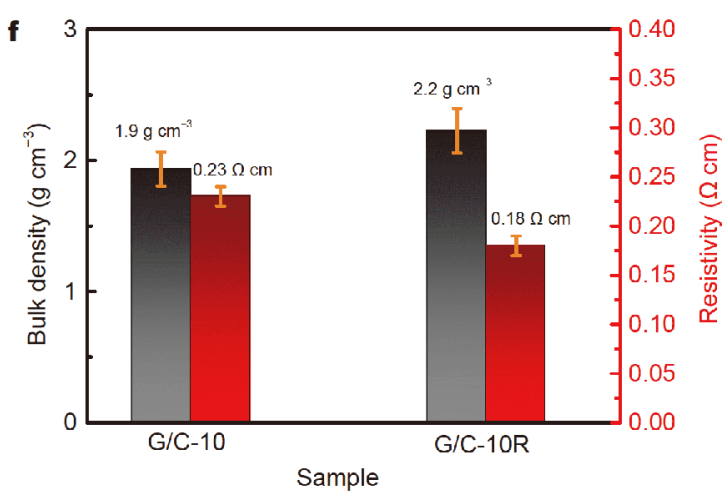

Figure 5 Surface SEM images of (a) G/C-10 and (b) G/C-10R coatings at 5000× magnification. Cross-sectional SEM images of (c) G/C-10 and (d) G/ C-10R coatings. (e) Pore diameter distribution of G/C-10 and G/C-10R coatings. (f) Bulk density and resistivity variation of G/C-10 and G/C-10R coatings. 
the GSs are distributed in the horizontal direction among the $\mathrm{CB}$ aggregates. After rolling compression, a more compact and smoother coating was obtained (Fig. 5b). The most likely pore size of the G/C-10 coating decreased from 50.2 to $32.3 \mathrm{~nm}$ (Fig. 5e), and the bulk density increased from 1.9 to $2.2 \mathrm{~g} \mathrm{~cm}^{-3}$ (Fig. 5f). Moreover, the sandwich structure becomes more obvious (Fig. 5d). Therefore, the compressed coating shows a much lower resistivity of $0.18 \pm 0.01 \Omega \mathrm{cm}$, reflecting a decrease of $21.7 \%$ (Fig. 5f). This resistivity is lower than that of other aqueous carbon-based inks [48-51] (Table 2). There are two reasons for this behavior. On the one hand, the contact area between the conductive fillers increases after rolling, and the conductive network becomes tighter. Within the conductive phase of the coatings, CB facilitates the conduction of electrons in the vertical direction, and graphene promotes the conduction of electrons in the horizontal plane. The 3D interlayer conduction contributes to enhancing the electronic properties [52]. On the other hand, the spacing between particles is reduced, making the hopping of electrons in the conductive filler easier and increasing the probability that electrons tunnel between nanofillers $[53,54]$.

Flexibility is the basic requirement for flexible devices; therefore, a cyclic bending test (video demo in Fig. S4) was conducted at a bending radius of $6 \mathrm{~mm}$ with homemade mechanical flexibility equipment, as shown in Fig. 6a. The resistance of the G/C-10R coating, derived from rolling compression of the G/C-10 coating $(4 \mathrm{~cm} \times$ $4 \mathrm{~cm}$, length $\times$ width) is 188,203 and $211 \Omega$ after 0,1500 and 3000 bending cycles, respectively. The normalized resistance $\left(R / R_{0}\right.$, resistance of the bent sample/initial resistance of the sample) is between 1 and 1.13 (Fig. 6e). In addition, part of the metal wire was replaced by a printed circuit, and was used to connect a light-emitting diode (LED) to a direct current (DC) power supply and printed circuit. The voltage was adjusted to $6 \mathrm{~V}$. The brightness of the LED did not change much when the conductive cir- cuit was unbended, bended and twisted, as shown in Fig. 6f-h (video demo in Fig. S5). These results indicate that the printed patterns show excellent mechanical flexibility that may be due to the high mechanical strength of the GSs and strong adhesion between the GSs and $\mathrm{CB}$.

To verify the practical application of the printed patterns, $\mathrm{CB} / \mathrm{GSs}$ coatings were used to produce luminescent bookmarks (video demo in Fig. S6) and membrane switches. LEDs were fixed to printed lines on a lightemitting bookmark (Fig. 6j) via conductive silver paste. The LEDs connected to the homemade bookmarks are bright at $6 \mathrm{~V}$. In addition, we fabricated a membrane switch with two printed lines $(50 \mathrm{~mm} \times 10 \mathrm{~mm} \times$ $0.01 \mathrm{~mm}$, length $\times$ width $\times$ thickness), as shown in Fig. $6 \mathrm{k}$. The two printed lines were separated by insulating spacers. Then, the membrane switch was connected to the circuits of an acoustical generator. Digital audio can be played or turned off by controlling the membrane switch (video demo in Fig. S7).

\section{CONCLUSIONS}

In summary, we have described a high-throughput and simple route for the well dispersion of GSs and CB in PAA by one-step ball milling. The water-based G/C-10 ink had suitable rheological characteristics for screen printing. By tuning the mass ratio of GSs to $\mathrm{CB}$, a coating comprising an optimal two-level network was obtained. In addition, the rough coating surface became compact and smooth after rolling compression, which eliminated the high roughness caused by the addition of GSs. Therefore, the resistivity of the G/C-10 coating decreased from $0.23 \pm 0.01$ to $0.18 \pm 0.01 \Omega \mathrm{cm}$. Moreover, the conductive coating had good mechanical flexibility after 3000 bending cycles, and the normalized resistance remained in the range of 1-1.13. Successful preparation of luminescent bookmarks and membrane switches by using the printed carbon-based conductive patterns reveals that

Table 2 Volume resistance comparison of aqueous carbon-based inks

\begin{tabular}{|c|c|c|c|c|}
\hline Solvents & Conductive fillers & Volume resistance $(\Omega \mathrm{cm})$ & Coating method & Reference \\
\hline $\mathrm{H}_{2} \mathrm{O}$ & $\mathrm{CB}$ & 71 & Screen printing & {$[51]$} \\
\hline Ethanol, EG, glycerol, water & $\mathrm{RGO}, \mathrm{CB}$ & 66.1 & Inkjet printing & {$[36]$} \\
\hline $\mathrm{H}_{2} \mathrm{O}$ & $\mathrm{CNF}$ & 1.8 & Vacuum filtration & {$[50]$} \\
\hline $\mathrm{H}_{2} \mathrm{O}$ & Nano carbons & 1.1 & Vacuum filtration & {$[48]$} \\
\hline $\mathrm{H}_{2} \mathrm{O}$ & MWCNTs & 0.64 & Inkjet printing & {$[49]$} \\
\hline $\mathrm{H}_{2} \mathrm{O}$ & G1600, CB & 0.18 & Screen printing & Our work \\
\hline
\end{tabular}

rGO: reduced graphene oxide; CB: carbon black; CNF: carbon nanofiber; MWCNTs: multiwalled carbon nanotubes; EG: ethylene glycol. 

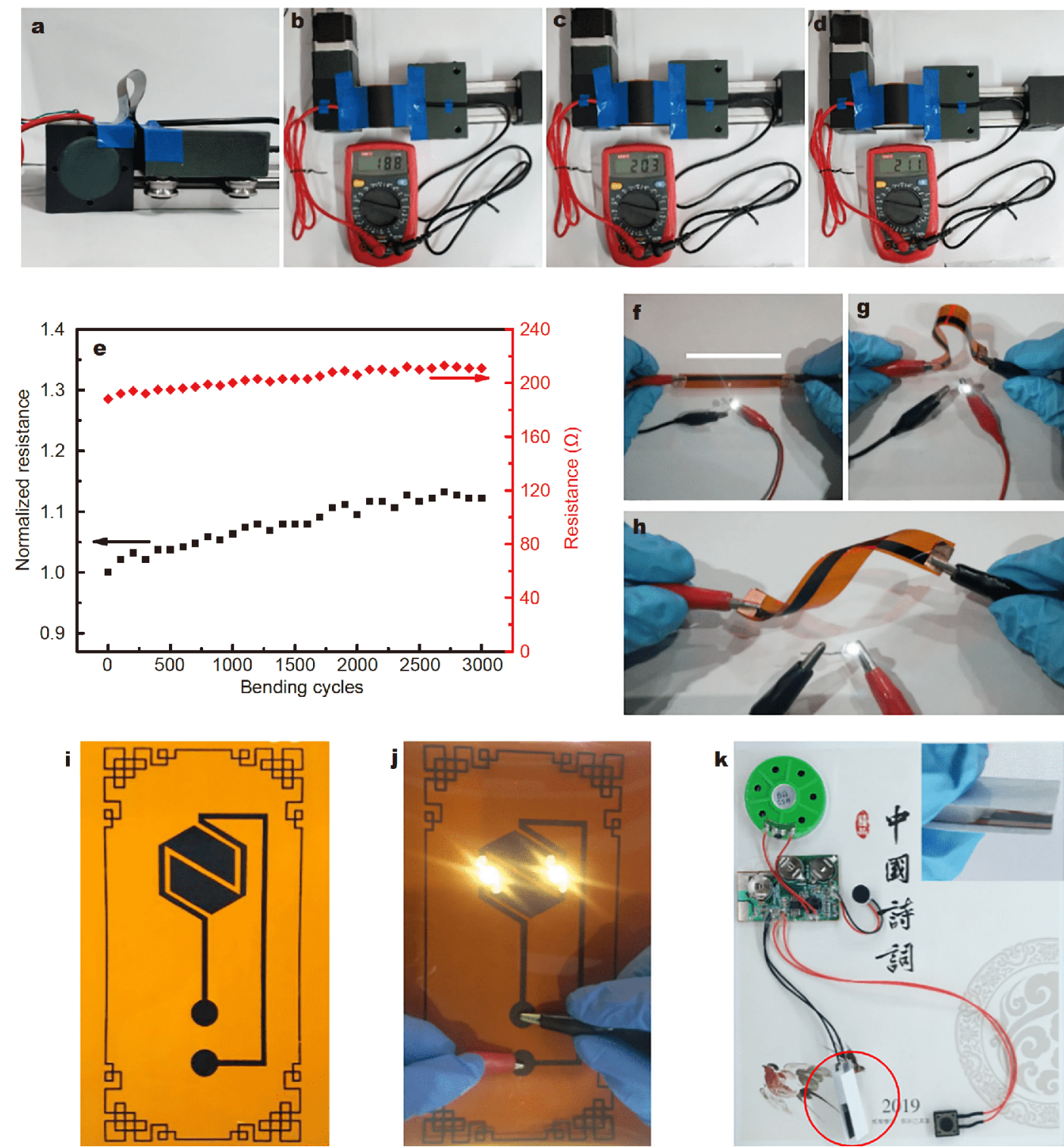

Figure 6 (a) Homemade mechanical flexibility test equipment. (b-d) The resistance values of the G/C-10R coating after bending 0 , 1500 and 3000 times. (e) The resistance and normalized resistance changes as a function of the bending cycle. The brightness of the LED when the printed circuit is in (f) unbended, (g) bended and (h) twisted conditions at $6 \mathrm{~V}$. Device demonstrations of printed patterns. (i) Screen-printed patterns of a luminous bookmark; (j) luminous bookmark at $6 \mathrm{~V}$. (k) Membrane switch made from printed circuits.

these water-based conductive inks have great potential in flexible printed electronics such as radio frequency identification systems and organic thin-film transistors.

\section{Received 22 August 2019; accepted 21 October 2019;} published online 15 November 2019

1 Singh R, Singh E, Nalwa HS. Inkjet printed nanomaterial based flexible radio frequency identification (RFID) tag sensors for the internet of nano things. RSC Adv, 2017, 7: 48597-48630

2 Wood V, Panzer MJ, Chen J, et al. Inkjet-printed quantum dot- polymer composites for full-color AC-driven displays. Adv Mater, 2009, 21: 2151-2155

3 Wu B, Zhang X, Huang B, et al. High-performance wireless ammonia gas sensors based on reduced graphene oxide and nanosilver ink hybrid material loaded on a patch antenna. Sensors, 2017, 17: 2070

4 Cinti S, Arduini F. Graphene-based screen-printed electrochemical (bio)sensors and their applications: Efforts and criticisms. Biosens Bioelectron, 2017, 89: 107-122

5 Secor EB, Dos Santos MH, Wallace SG, et al. Tailoring the porosity and microstructure of printed graphene electrodes via polymer phase inversion. J Phys Chem C, 2018, 122: 13745-13750 
6 Secor EB, Gao TZ, Dos Santos MH, et al. Combustion-assisted photonic annealing of printable graphene inks via exothermic binders. ACS Appl Mater Interfaces, 2017, 9: 29418-29423

7 Naik AR, Kim JJ, Usluer Ö, et al. Direct printing of graphene electrodes for high-performance organic inverters. ACS Appl Mater Interfaces, 2018, 10: 15988-15995

8 Sirringhaus H, Kawase T, Friend RH, et al. High-resolution inkjet printing of all-polymer transistor circuits. Science, 2000, 290: 2123-2126

9 Huang X, Leng T, Zhang X, et al. Binder-free highly conductive graphene laminate for low cost printed radio frequency applications. Appl Phys Lett, 2015, 106: 203105

10 Huang X, Leng T, Zhu M, et al. Highly flexible and conductive printed graphene for wireless wearable communications applications. Sci Rep, 2016, 5: 18298

11 Tran TS, Dutta NK, Choudhury NR. Graphene inks for printed flexible electronics: Graphene dispersions, ink formulations, printing techniques and applications. Adv Colloid Interface Sci, 2018, 261: 41-61

12 Kamyshny A, Magdassi S. Conductive nanomaterials for printed electronics. Small, 2014, 10: 3515-3535

13 Secor EB, Gao TZ, Islam AE, et al. Enhanced conductivity, adhesion, and environmental stability of printed graphene inks with nitrocellulose. Chem Mater, 2017, 29: 2332-2340

$14 \mathrm{Hu}$ G, Kang J, Ng LWT, et al. Functional inks and printing of twodimensional materials. Chem Soc Rev, 2018, 47: 3265-3300

15 Barragán-Iglesias $\mathrm{P}$, Lou TF, Bhat VD, et al. Inhibition of poly(A)binding protein with a synthetic RNA mimic reduces pain sensitization in mice. Nat Commun, 2018, 9: 10-38

16 Zhao J, Song M, Wen C, et al. Microstructure-tunable highly conductive graphene-metal composites achieved by inkjet printing and low temperature annealing. J Micromech Microeng, 2018, 28: 035006

17 Magdassi S, Grouchko M, Kamyshny A. Copper nanoparticles for printed electronics: Routes towards achieving oxidation stability. Materials, 2010, 3: 4626-4638

18 Rösch R, Tanenbaum DM, Jørgensen M, et al. Investigation of the degradation mechanisms of a variety of organic photovoltaic devices by combination of imaging techniques-the ISOS-3 interlaboratory collaboration. Energy Environ Sci, 2012, 5: 6521-6540

$19 \mathrm{Su} \mathrm{C}, \mathrm{Bu} \mathrm{X}, \mathrm{Xu} \mathrm{L}$, et al. A novel $\mathrm{LiFePO}_{4} /$ graphene/carbon composite as a performance-improved cathode material for lithium-ion batteries. Electrochim Acta, 2012, 64: 190-195

20 Murr LE. Nanoparticulate materials in antiquity: The good, the bad and the ugly. Mater Charact, 2009, 60: 261-270

21 Khandavalli S, Park JH, Kariuki NN, et al. Rheological investigation on the microstructure of fuel cell catalyst inks. ACS Appl Mater Interfaces, 2018, 10: 43610-43622

22 Youssry M, Madec L, Soudan P, et al. Non-aqueous carbon black suspensions for lithium-based redox flow batteries: rheology and simultaneous rheo-electrical behavior. Phys Chem Chem Phys, 2013, 15: 14476-14486

23 Jo Y, Kim JY, Kim SY, et al. 3D-printable, highly conductive hybrid composites employing chemically-reinforced, complex dimensional fillers and thermoplastic triblock copolymers. Nanoscale, 2017, 9: 5072-5084

24 Youssry M, Kamand FZ, Magzoub MI, et al. Aqueous dispersions of carbon black and its hybrid with carbon nanofibers. RSC Adv, 2018, 8: 32119-32131

25 Latil S, Henrard L. Electric field effect in atomically thin carbon films. Science, 2004, 306: 666-669

26 Raccichini R, Varzi A, Passerini S, et al. The role of graphene for electrochemical energy storage. Nat Mater, 2014, 14: 271-279

27 Hernandez Y, Lotya M, Rickard D, et al. Measurement of multicomponent solubility parameters for graphene facilitates solvent discovery. Langmuir, 2010, 26: 3208-3213

28 Novoselov KS, Fal'ko VI, Colombo L, et al. A roadmap for graphene. Nature, 2012, 490: 192-200

29 Arapov K, Jaakkola K, Ermolov V, et al. Graphene screen-printed radio-frequency identification devices on flexible substrates. Phys Status Solidi RRL, 2016, 10: 812-818

30 Arapov K, Rubingh E, Abbel R, et al. Conductive screen printing inks by gelation of graphene dispersions. Adv Funct Mater, 2016, 26: $586-593$

31 Hyun WJ, Secor EB, Hersam MC, et al. High-resolution patterning of graphene by screen printing with a silicon stencil for highly flexible printed electronics. Adv Mater, 2015, 27: 109-115

32 Zabek D, Seunarine K, Spacie C, et al. Graphene ink laminate structures on poly(vinylidene difluoride) (PVDF) for pyroelectric thermal energy harvesting and waste heat recovery. ACS Appl Mater Interfaces, 2017, 9: 9161-9167

$33 \mathrm{Xu} \mathrm{Y,} \mathrm{Hennig} \mathrm{I,} \mathrm{Freyberg} \mathrm{D,} \mathrm{et} \mathrm{al.} \mathrm{Inkjet-printed} \mathrm{energy} \mathrm{storage}$ device using graphene/polyaniline inks. J Power Sources, 2014, 248: $483-488$

34 Wang R, Li W, Liu L, et al. Carbon black/graphene-modified aluminum foil cathode current collectors for lithium ion batteries with enhanced electrochemical performances. J Electroanal Chem, 2019, 833: 63-69

35 Cai J, Hu N, Wu L, et al. Preparing carbon black/graphene/PVDFHFP hybrid composite films of high piezoelectricity for energy harvesting technology. Compos Part A-Appl Sci Manufact, 2019, 121: 223-231

36 Ji A, Chen Y, Wang X, et al. Inkjet printed flexible electronics on paper substrate with reduced graphene oxide/carbon black ink. J Mater Sci-Mater Electron, 2018, 29: 13032-13042

37 Chen Y, Zhou L, Wei J, et al. Direct ink writing of flexible electronics on paper substrate with graphene/polypyrrole/carbon black ink. J Elec Materi, 2019, 48: 3157-3168

38 Hua $\mathrm{C}, \mathrm{Li} \mathrm{X}$, Shen $\mathrm{L}$, et al. Influence of co-solvent hydroxyl group number on properties of water-based conductive carbon pastes. Particuology, 2017, 33: 35-41

39 Lin X, Zhang K, Li K, et al. Dependence of rheological behaviors of polymeric composites on the morphological structure of carbonaceous nanoparticles. J Appl Polym Sci, 2018, 135: 46416

40 Chakraborty G, Gupta A, Pugazhenthi G, et al. Facile dispersion of exfoliated graphene/PLA nanocomposites via in situ polycondensation with a melt extrusion process and its rheological studies. J Appl Polym Sci, 2018, 135: 46476

41 Mao T, Tang Y, Zhang Y, et al. Carbon nanotubes/polyaniline nanocomposite coatings: Preparation, rheological behavior, and their application in paper surface treatment. J Appl Polym Sci, 2018, 135: 46329

42 Eom Y, Kim F, Yang SE, et al. Rheological design of 3D printable all-inorganic inks using BiSbTe-based thermoelectric materials. J Rheology, 2019, 63: 291-304

43 Wang S, Zhong Y, Fang H. Deformation characteristics of a single droplet driven by a piezoelectric nozzle of the drop-on-demand inkjet system. J Fluid Mech, 2019, 869: 634-645

44 Kitsomboonloha R, Morris SJS, Rong X, et al. Femtoliter-scale patterning by high-speed, highly scaled inverse gravure printing. 
Langmuir, 2012, 28: 16711-16723

45 Taroni M, Breward CJW, Howell PD, et al. Boundary conditions for free surface inlet and outlet problems. J Fluid Mech, 2012, 708: $100-110$

46 Su FY, You C, He YB, et al. Flexible and planar graphene conductive additives for lithium-ion batteries. J Mater Chem, 2010, 20: 9644-9650

47 Ruschau GR, Yoshikawa S, Newnham RE. Resistivities of conductive composites. J Appl Phys, 1992, 72: 953-959

48 Hof F, Kampioti K, Huang K, et al. Conductive inks of graphitic nanoparticles from a sustainable carbon feedstock. Carbon, 2017, 111: $142-149$

49 Jason Jan C, Walton MD, McConnell EP, et al. Carbon black thin films with tunable resistance and optical transparency. Carbon, 2006, 44: 1974-1981

$50 \mathrm{Lu} \mathrm{H}$, Liu Y, Gou J, et al. Electroactive shape-memory polymer nanocomposites incorporating carbon nanofiber paper. Int J Smart Nano Mater, 2010, 1: 2-12

51 Pahalagedara LR, Siriwardane IW, Tissera ND, et al. Carbon black functionalized stretchable conductive fabrics for wearable heating applications. RSC Adv, 2017, 7: 19174-19180

52 Jimenez MJM, de Oliveira RF, Bufon CCB, et al. Enhanced mobility and controlled transparency in multilayered reduced graphene oxide quantum dots: a charge transport study. Nanotechnology, 2019, 30: 275701

53 Yu S, Wang X, Xiang H, et al. 1-D polymer ternary composites: Understanding materials interaction, percolation behaviors and mechanism toward ultra-high stretchable and super-sensitive strain sensors. Sci China Mater, 2019, 62: 995-1004

54 Zhang Q, Xu Y, Yang Y, et al. Conductive mechanism of carbon black/polyimide composite films. J Polymer Eng, 2018, 38: 147-156

Acknowledgements This research was supported by the Scientific and Technological Key Project of Shanxi Province (MC2016-04 and MC2016-08), Natural Science Foundation of Shanxi Province (201801D221156), DNL Cooperation Fund of CAS (DNL180308), Science and Technology Service Network Initiative of CAS (KFJ-STSZDTP-068), and Youth Innovation Promotion Association of CAS.

Author contributions Yang X, Li XM and Chen CM conceived the project. Yang X performed the preparation of conductive inks, printing of patterns, characterization of inks and patterns as well as the demonstration of luminescent and membrance switch. Li XM set up the mechanical flexibility equipment. Kong QQ, Liu Z and Xie LJ supervised the project. Jia H contributed the photographs. Yang X, Li XM, Chen JP and Liu YZ wrote and amended the paper.

Conflict of interest The authors declare no conflict of interest.

Supplementary information Supporting data are available in the online version of the paper.

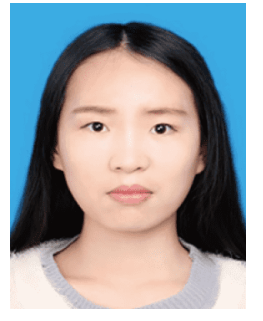

Xiao Yang received her Bachelor of Engineering degree from Shanxi University of Science and Technology in 2017. She is currently pursuing a Master's degree under the supervision of Prof. Chengmeng Chen at the Institute of Coal Chemistry, Chinese Academy of Sciences. Her research involves the design and synthesis of conductive inks for flexible printed electronics applications.

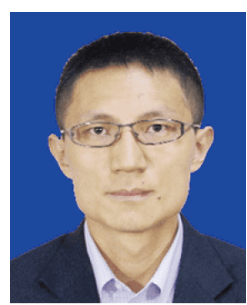

Chengmeng Chen received his $\mathrm{PhD}$ in materials science in 2012 from the Institute of Coal Chemistry, Chinese Academy of Sciences. Currently, he is a professor and Vice Director of the Key Laboratory of Carbon Materials, Institute of Coal Chemistry, Chinese Academy of Sciences. Dr. Chen is committed to the research and development of advanced carbon materials and energy storage devices.

\section{一步球磨法制备石墨烯/炭黑水性导电油墨应用} 于柔性电子器件

杨晓 ${ }^{1,2}$, 李晓明 ${ }^{1}$, 孔庆强 ${ }^{1,2}$, 刘卓 ${ }^{1}$, 陈景鹏 ${ }^{1,2}$, 贾辉 ${ }^{1,2}$, 刘燕珍 ${ }^{1}$, 谢莉婧 ${ }^{1}$, 陈成猛 ${ }^{1 *}$

摘要 本文以石墨烯和炭黑作为导电填料, 通过一步球磨法制备了 高导电的水性碳系油墨. 当石墨烯质量分数为 $10 \%$ 时, 复合油墨具 有最优的流变学性能; 在丝网印刷中, 该油墨在聚酰亚胺基底上形 成均匀致密的涂层. 在微观上, 涂层具有夹层三维网络结构, 电阻率 为 $0.23 \pm 0.01 \Omega \mathrm{cm}\left(92 \pm 4 \Omega \mathrm{sq}^{-1}, 25 \mu \mathrm{m}\right)$, 是纯炭黑涂层电阻率 $(0.77 \pm 0.01 \Omega \mathrm{cm})$ 的 $30 \%$. 辊压处理后, 电阻率降至 $0.18 \pm 0.01 \Omega \mathrm{cm}$ $\left(72 \pm 4 \Omega \mathrm{sq}^{-1}, 25 \mu \mathrm{m}\right)$, 且涂层具有良好的机械柔性, 经过 3000 次循 环弯折试验后电阻仅增加 $12 \%$. 最后, 我们将石墨烯/炭黑复合涂层 作为柔性导体, 成功组装了发光书签和薄膜开关, 表明该涂层在柔 性电子器件大规模生产中具有巨大的潜力. 